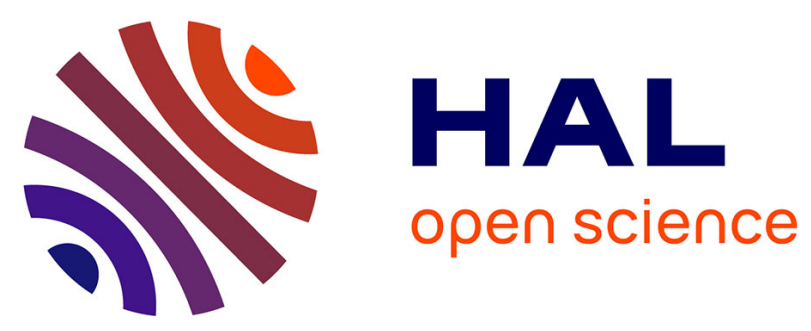

\title{
Synergistic or Antagonist Effects of Different UV Ranges Analyzed by the Combination Index: Application to DNA Photoproducts
}

\author{
Thierry Douki, Arnaud Buhot
}

\section{- To cite this version:}

Thierry Douki, Arnaud Buhot. Synergistic or Antagonist Effects of Different UV Ranges Analyzed by the Combination Index: Application to DNA Photoproducts. Photochemistry and Photobiology, In press, 10.1111/php.13528 . hal-03356359v2

HAL Id: hal-03356359

https://hal.science/hal-03356359v2

Submitted on 11 Oct 2021

HAL is a multi-disciplinary open access archive for the deposit and dissemination of scientific research documents, whether they are published or not. The documents may come from teaching and research institutions in France or abroad, or from public or private research centers.
L'archive ouverte pluridisciplinaire HAL, est destinée au dépôt et à la diffusion de documents scientifiques de niveau recherche, publiés ou non, émanant des établissements d'enseignement et de recherche français ou étrangers, des laboratoires publics ou privés. 


\title{
Synergistic or Antagonist Effects of Different UV Ranges
}

\section{Analyzed by the Combination Index: Application to DNA}

\section{Photoproducts}

\author{
Thierry Douki* and Arnaud Buhot
}

Univ. Grenoble Alpes, CEA, CNRS, IRIG, SyMMES, F-38000 Grenoble

Corresponding authors: Thierry Douki

Email: thierry.douki@,cea.fr

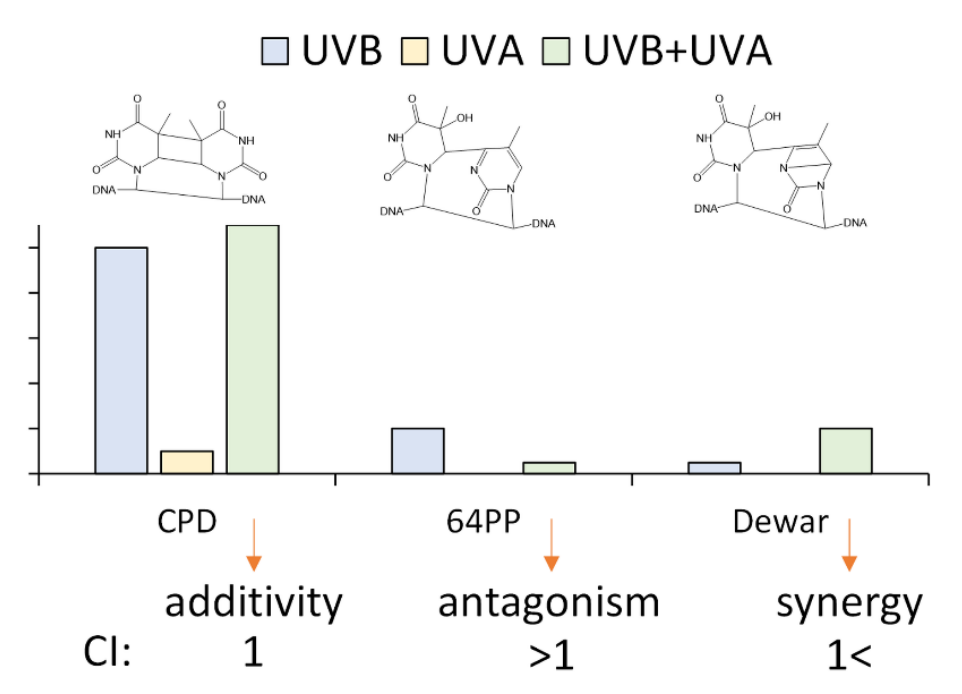

Combination index (CI) is a data analysis tool reflecting antagonistic or inhibitory effects of complex exposures. Applied to DNA photochemistry, CI could unambiguously demonstrate the additive effects of UVB and UVA on the formation of cyclobutane pyrimidine dimers (CPD). CI also clearly assessed the enhanced yield of Dewar valence isomers (Dewar) at the expense of that of (6-4) photoproducts (64PP) upon exposure to combinations of UVB and UVA. These examples illustrate the usefulness of the CI strategy in photobiology. 


\section{ABSTRACT} Keywords: data modeling, co-exposure, action spectra, non-additive effects, DNA damage

Photobiological effects are known to greatly depend on the wavelength of the incident photons that define the nature of the activated chromophores. A growing number of experimental data show that considering the effect of complex light sources as a sum of the effects of monochromatic exposures can be misleading. Indeed, the combined exposure to several wavelength ranges may modulate photobiological responses or even induce novel processes. These observations are similar to a well-known topic in chemical toxicology: the non-additivity of effects in mixtures where either antagonism or synergy are often observed. In the present work, we investigated if a data analysis tool first developed for studying non-additivity in mixtures of drugs, the combination index, could be applied to photobiological processes. We chose to work on the formation of UV-induced DNA photoproducts where additive, antagonist and synergistic effects take place simultaneously. In addition to this application, we worked on the mathematical bases of the concept in order to broaden its applicability to phenomena exhibiting various dose-response patterns. We also addressed the question of the evaluation of the error on the determination of the combination index. 
The activation and the intensity of photobiological responses are obviously dependent on the fluence of the radiation but also of the wavelength of the incident photons. This has been realized for a long time and is classically taken into account by the use of action spectra (1-4), which report the wavelength-dependent variation of the intensity of an effect per dose unit. By convolution with the emission spectrum of a source of interest, action spectra provide an estimation of the biological effect under specific experimental conditions. However, the use of action spectra is based on an additivity principle that is not always relevant in photobiology. Some photobiological effects may result from the consecutive impact of two different wavelength ranges. In this case, a synergy is observed. Conversely, there are cases where the response triggered by a wavelength range is decreased by another, leading to an antagonism. Examples can be found in the photomorphogenesis of plants that is activated and inhibited at two different wavelengths, (5) or in bacteria where sunlight simultaneously induces DNA damage and activates photolyase repair enzymes $(6,7)$. As we recently reviewed, similar non-additive effects have been reported in humans and in mammalian cells (8). Immunosuppression is induced by both UVB and UVA but UVA may counteract the effect of UVB (9-11). Similarly, the extent of apoptosis depends on the ratio between the two wavelength ranges upon co-exposure to UVB and UVA (12). More recently, UVA was reported to modulate the cytotoxicity of UVB (13). Other examples concern the induction of oxidative stress. UVA was found to promote the formation of lipofuscin that is a visible light photosensitizer (14). Similarly, some UVB photoproducts of tryptophan like N-formylkynurenine are UVA-photosensitizers (15-17). Last, in the field of DNA damage, pyrimidine (6-4) pyrimidone photoproducts (64PPs) are produced by UVB radiation in DNA and efficiently converted into their Dewar valence isomers (Dewars) by UVA photons $(18,19)$. 
61 The non-additive effects of different wavelength ranges represent thus a key issue in the prediction and understanding of photobiological effects. Unfortunately, they are not frequently taken into consideration or only on a very limited number of applied doses. This last point is important because some effects can be additive within a dose range and antagonist or synergistic in another. The purpose of the present work is thus to provide a methodology for the identification of non-additivity effects in photobiology under the largest set of conditions possible with the most reasonable amount of experimental work. Ideas of experimental design and data treatment for this problem can be found in the field of the pharmaceutical and toxicological effects of chemicals, where examples of non-additive responses to the combination of organic or inorganic compounds are countless. The question of the non-additive effects has been put forward for many years with the notions of exposome and cocktail effects (20). Several mathematical models have been designed to analyze experimental data and establish the occurrence, or not, of synergy or antagonism in the impact of mixtures (21-23).

Among them, the combination index (CI) approach proposed by Chou (24-27) is particularly attractive as it combines the a priori definition of an optimal experimental plan with a strict mathematical approach for the identification of antagonist or synergistic effect. This strategy was designed for the optimization of the efficiency of combinations of drugs. It was very successful in various fields. More than 1500 references to the combination index could be retrieved, for a large part in the field of cancer treatment but also in studies on HIV or in toxicology investigations. The most complete review on the combination index (25) has received more than 4000 citations. The mathematical model designed by Chou is derived from the mass action law and is well suited to study the interaction of a drug with a physiological target $(25,27)$. Extensive mathematical analysis of the underlying phenomena led Chou and his co-workers to propose a rather simple equation describing pharmaco-kinetics interaction, the 
medium effect equation (MEE). The same mathematical framework led to the design of the CI, a convenient tool to study antagonist and synergistic effects.

As it originates from the mass action law, the MEE can precisely model data ranging between 0 and 1 (or 0 and $100 \%$ ) with dose-dependent curves of the responses exhibiting sigmoidal or hyperbolic shapes. In addition, the MEE requires only two fitting parameters thus limiting the required dose-response experiments for their determination. Determining the MEE is a requisite to the calculation of the CI. The value of the CI shows whether the effects of a mixture are additive $(C I=1)$, antagonist $(C I>1)$ or synergistic $(C I<1)$. The strategy underlying the $C I$ approach is illustrated on a theoretical example on Scheme 1. In the framework designed by Chou and co-workers, results are expressed as the affected fraction $f a$, namely the yield of a process. Experiments are first performed with the two studied chemical or physical factors alone in a series of doses or concentrations. The parameters of the MEE are calculated for each set of data by a Log-Log transformation. More mathematical details are provided in the "Data Modeling" part below. The $C I$ can then be calculated for any combination of the two factors. Obviously, it is of outmost importance to determine the $C I$ over a wide variety of mixture compositions, which drive the extent of deviation from additivity. Therefore, $C I$ investigations include a third experiment in which the two factors are applied simultaneously in different concentrations but with a constant ratio. The global effect $f x$ of each of these combinations $D 1+D 2$ is determined. The MEE for the two factors are then used to determine the concentration of the dose $D x 1$ and $D x 2$ of pure factors 1 and 2 necessary to induce the same effect $f x$ as the mixture. The $C I$ is defined as $\frac{D 1}{D x 1}+\frac{D 2}{D x 2}$. Additivity is shown by a value of $C I$ of 1. This can be illustrated by an example with two factors equally efficient at inducing a response (same MEE). If a 1:1 mixture of factors 1 and 2 at a dose $D$ induces a response $f x, D x 1$ value will be $2 D$ (because factor 1 alone is as efficient as factor 2 in the mixture). The same will be obtained for factor 2 . Therefore, $C I=\frac{D}{2 D}+\frac{D}{2 D}=\frac{1}{2}+\frac{1}{2}=1$. Similar calculations can be made 
110 when factors 1 and 2 are not equally efficient. Keeping this example of equally efficient factors

1111 and 2, it may be observed in other cases that the dose of pure factor 1 or pure factor 2 required

112 to observe a response $f x$ is less than $2 D$. In this case the $\frac{D}{D x}$ terms in $C I$ are larger than $\frac{1}{2}$ and

113 consequently $C I>1$. This correspond to a situation where a larger amount of the two factors are

114 necessary in mixture than as pure products to observe a same effect. This is typically the case

115 of an antagonist mixture effect. An opposite calculation can be made for synergy effects that 116 correspond to $C I<1$.

(a)

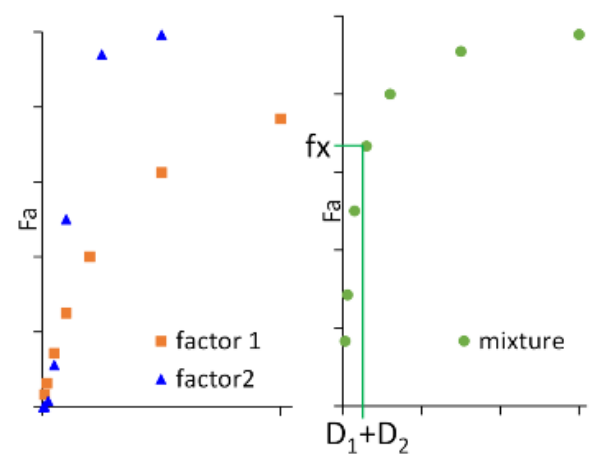

(c)

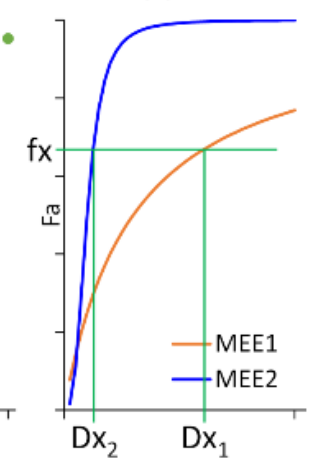

(d)

$C I=\frac{D 1}{D x 1}+\frac{D 2}{D x 2}$

$\mathrm{Cl}=1 \rightarrow$ additivity

$\mathrm{Cl}>1 \rightarrow$ Inhibition

$\mathrm{Cl}<1 \rightarrow$ synergy
117

Scheme 1: Principle of the determination of the combination index CI. (a) The amplitude of the studied effect fa is determined for factors 1 and 2 applied alone on a range of concentrations that can be different for the two factors. (b) Experiments are performed with mixtures of factors 1 and 2 exhibiting a constant ratio $D_{1} / D_{2}$. The $C I$ can be determined for each of the mixtures. Those have a concertation $D_{1}+D_{2}$ and induce an effect $f x$. (c) Data from (a) are fitted by the MEE. The latter equation provides the values of the concentration of pure factors $1\left(D x_{1}\right)$ and $2\left(D x_{2}\right)$ leading to the effect $f x$. (d) The CI is calculated and the conclusion on additivity, synergy or antagonism is deduced.

In the present work, we aimed at providing a proof-of-concept that the $C I$ approach could be used in photobiology. For this purpose, we applied the $C I$ formalism to the well-known UV photochemistry of DNA (Scheme 2), and in particular to the formation of pyrimidine dimers (28-30). These photoproducts arise, upon exposure to UVB, from the cycloaddition of the C5-C6 double bonds of adjacent pyrimidine bases or from the addition of the C5-C6 double 
132 bond of the 3 '-end pyrimidine to the $\mathrm{C} 4$ carbonyl or imine group of the 5 '-end pyrimidine. The

133 respective products are cyclobutane pyrimidine dimers (CPDs) and 64PPs. CPDs are also

134 produced by UVA, although in much lower amounts than by UVB. A specific feature of 64PPs

135 is that their pyrimidone moiety can absorb a second UV photon and undergo photoisomerization

136 into Dewars. In isolated photoproducts, this photoreaction is most efficient at approximately

$137320 \mathrm{~nm}(31,19,32,33)$, but this maximum is shifted towards the UVA region (18) within DNA

138 because of the presence of overwhelming amounts of normal bases that shield UVB photons

139 (34). DNA photochemistry thus offers examples of additive (CPDs), synergistic (Dewars) and

140 antagonist (64PPs) effects between UVB and UVA. In addition, the yield of photoproducts

141 cannot be more than $100 \%$. The latter observation is reinforced by the fact that experiments

142 are always performed at a low modification rate that mimics real life situations. In that respect,

143 these data are well suited for application of the MEE. We also used them to explore the use of

144 other modeling equations in place of the MEE. Indeed, the latter function does not reproduce

145 all possible dose-course shapes of biological responses, which can be more complex than a drug

146 / target interaction. In addition, numerous effects do not range between 0 and 1, and can exhibit

147 no theoretical limit.
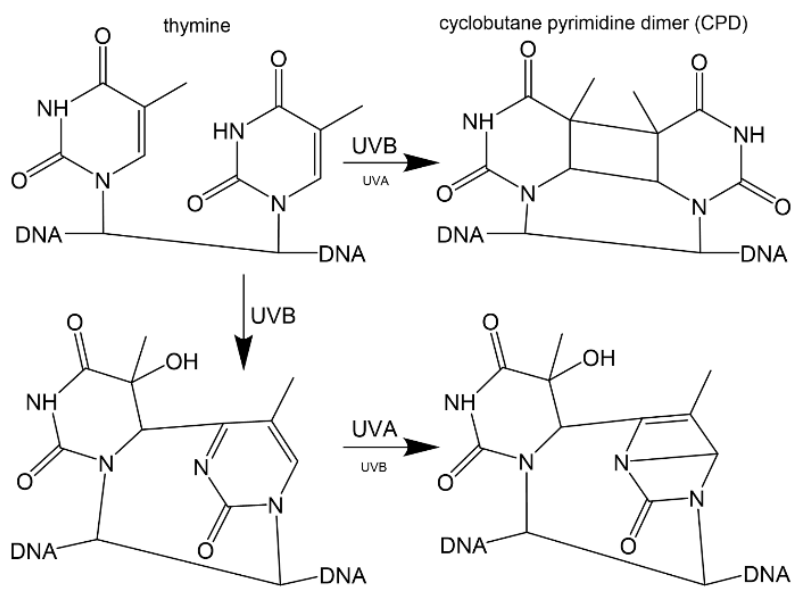

(6-4) photoproduct (64PP)

Dewar valence isomer (Dewar)

149 Scheme 2: Structure of the dimeric pyrimidine photoproducts induced in DNA by UVB and UVA. The photoreactions are described for two thymines but similar products can be formed 
153 Irradiations were performed with high-grade calf thymus DNA (Sigma, Saint Quentin Fallavier,

154 France). A stock solution at approximately $1 \mathrm{mg} \mathrm{mL}^{-1}$ was prepared in deionized water (Milli

155 Q) and desalted by dialysis for $24 \mathrm{~h}$ in water with repeated changing of the water bath. The 156 solution was then diluted in an aqueous $50 \mathrm{mM} \mathrm{NaCl}$ solution at a DNA concentration of 50 $157 \mu \mathrm{g} \mathrm{mL}^{-1}$. It was then exposed to UV radiation in $1 \times 1 \times 4 \mathrm{~cm}$ fluorimeter quartz cell (Hellma 158 Analytics, Munich, Germany). The irradiation set-up consisted of three lamps. A 2x15W and a $15915 \mathrm{~W}$ UVA lamps were placed facing perpendicular sides of the sample. Both lamps carried

160 T15-L Vilber-Lourmat tubes (Marne-La Vallée, France) exhibiting an emission spectrum ranging from 320 to $380 \mathrm{~nm}$ with a maximum at $365 \mathrm{~nm}$. A UVB lamp fitted with a $15 \mathrm{~W}$ T15-M tube exhibiting an emission centered at $312 \mathrm{~nm}$ (Vilber-Lourmat, Marne-La Vallée, France) was placed on the opposite side of the $2 \times 15$ W UVA lamp. The UVA fluences were determined with a Model 5.7 Solar meter (Solar Light, Glenside, PA). The UVB dosimetry was carried out with a VLX 3W radiometer (Vilber Lourmat, Marne-la-Vallée, France) fitted with a $312 \mathrm{~nm}$ probe. The sample holder could accommodate filters and was placed on a magnetic stirrer for a constant homogenization of the sample during irradiation. For irradiation, the quartz cell was loaded with $2.5 \mathrm{~mL}$ of DNA solution and, except for experiment 3 (see below), $200 \mu \mathrm{L}$ aliquot

169 fractions were collected after increasing exposure times.

170 Four series of experiments were performed with different designs. Only the UVB and the $2 \times 15$

171 W UVA lamps used in the three first series while the 3 lamps were used in the last.

172 1) A filter with cut-off at $320 \mathrm{~nm}$ (Schott, Colombes, France) was placed in front of the samples in the UVA beam. The fluences were constant and set at $0.13 \mathrm{~kJ} \mathrm{~m}^{-2} \mathrm{~min}^{-1}$ for UVB and $2.36 \mathrm{~kJ} \mathrm{~m}^{-2} \mathrm{~min}^{-1}$ for UVA. Exposure times ranged between 1 and $90 \mathrm{~min}$, and the experiments were repeated twice. 
2) The fluence of the UVA source (with a cut-off filter at $320 \mathrm{~nm}$ ) was left constant at 0.65 $\mathrm{kJ} \mathrm{m}^{-2} \min ^{-1}{ }^{1}$. The position of the UVB lamp (with a cut-off filter at $295 \mathrm{~nm}$, Schott, Colombes, France) was changed to provide fluences of either $0.20,0.16,0.12,0.09$ or $0.03 \mathrm{~kJ} \mathrm{~m}^{-2} \mathrm{~min}^{-1}$. Irradiations were performed once under each UVB conditions with exposure times ranging between 4 and $64 \mathrm{~min}$. Samples were analyzed once except that

3) UVB and UVA fluences (with cut-off filters at 295 and $320 \mathrm{~nm}$, respectively) were kept constant at 0.08 and $0.73 \mathrm{~kJ} \mathrm{~m}^{-2} \mathrm{~min}^{-1}$, respectively. Five irradiation protocols were used. DNA samples $(2 \mathrm{~mL})$ were irradiated for either 5, 10, 20 or $40 \mathrm{~min}$. Three aliquot fractions $(200 \mu \mathrm{L})$ were collected from each of these UV-exposed solutions. The three first protocols involved either UVA, UVB or UVB and UVA simultaneously. In the fourth design, samples were exposed for $5 \mathrm{~min}$ to pure UVA and then for $5 \mathrm{~min}$ to UVB. The experiment was repeated with consecutive exposures to UVA and UVB lasting $10+10,20+20$ or $40+40 \mathrm{~min}$. The fifth protocol was similar except that UVB was applied before UVA. All these experiments were referred to as UVA, UVB, UVB+UVA, UVA $\rightarrow$ UVB and UVB $\rightarrow$ UVA. Each irradiation was performed once but all collected samples were analyzed three times and the obtained results were averaged before data analysis.

4) The three lamps were used with bandpass filters of $310 \pm 5 \mathrm{~nm}$ for UVB, $340 \pm 13 \mathrm{~nm}$ for the $1 \times 15 \mathrm{~W}$ UVA and $386 \pm 13.5 \mathrm{~nm}$ for $2 \times 15 \mathrm{~W}$ UVA. All bandpass filters were obtained from Edmund Optics (Lyon, France). The respective fluences were 0.0048, 0.036 and $0.045 \mathrm{~kJ} \mathrm{~m}^{-2} \mathrm{~min}^{-1}$, respectively. Irradiation lasted 30, 60, 90 and $120 \mathrm{~min}$. The experiment was repeated three times. 
Following irradiation, DNA was precipitated from the samples by addition of $\mathrm{NaCl}$ and cold ethanol. The level of photoproducts was then determined as previously reported (35). The DNA samples were enzymatically hydrolyzed by phosphodiesterases, DNases and alkaline phosphate. The resulting solutions were analyzed by high performance liquid chromatography coupled to tandem mass spectrometry.

\section{DATA MODELING}

All calculations were performed in Microsoft Excel spread sheets in order to copy and paste data directly from the software of the LC-MS/MS system. We determined the values of the parameters of the MEE (Dm and m), of the Dx and of CI using the method described by Chou $(25,27)$. We used Compusyn (ComboSyn Incorporated) on a subset of data to check that our calculations were correct. Excel sheets were also used for the calculation of the CI using other equations with the first set of experimental results. Values of the coefficients of the regressions and of their error were obtained from Origin (OriginLab Corporation, MA). For the calculation

213 of the propagation of errors, we used the classical method stating that if a measure $y$ is a function

214 of a parameter $\mathrm{x}$, then $\Delta \mathrm{y}=\left|\left(\frac{\mathrm{d} f}{\mathrm{dx}}\right)\right| \times \Delta \mathrm{x}$, where $\Delta \mathrm{y}$ and $\Delta \mathrm{x}$ are the errors on $\mathrm{y}$ and $\mathrm{x}$, respectively.

215 When y depends on more than one parameter, the absolute values of all errors are added: $\Delta y=$ $216\left|\left(\frac{d f}{d \times 1}\right)\right| \times \Delta \times 1+\left|\left(\frac{d f}{d \times 2}\right)\right| \times \Delta \times 2\left|\left(\frac{d f}{d \times 3}\right)\right| \times \Delta x 3+\cdots$

\section{Medium effect equation}

218 The MEE used in the original work by Chou et al. (25) is the following:

$$
\text { (1) } \frac{f a}{(1-f a)}=\left(\frac{D}{D m}\right)^{m}
$$

220 where $f a$ is the response referred to as affected fraction in the framework of drug/target 221 interactions, $D$ the dose, $D m$ the dose leading to a $50 \%$ of the maximal effect, and $m$ a coefficient 
reflecting the shape of the curve $(m>1 \rightarrow$ hyperbolic; $m=1 \rightarrow$ sigmoidal; $m<1 \rightarrow$ "flat" 223 sigmoidal).

224 The $D m$ and $m$ parameters were determined from the experimental data by linear regression of 225 Log-Log curve

(2) $\log \left(\frac{f a}{1-f a}\right)=f(\log (D))=a \times \log (D)+b=m \times \log (D)-m \times \log (D m)$

$227 m$ is thus the slope $a$ and $D m$ is $10^{-\frac{a}{b}}$.

228 Calculation of the $C I$ requires the determination of the dose $D x$ of each factor leading to the 229 same effect $f x$ than a given mixture. In other words, it is necessary to invert the MEE equation 230 (1) to determine $D x$ for a measured effect $f x$, a known value of $\frac{f a}{1-f a}$ :

$231 \quad$ (3) $D x=D m \times[f x]^{1 / m}$

233 The error on $D x(\Delta D x)$ can be inferred from that on $m$ and $D m$. The coefficients $m$ and $D m$ were 234 obtained by linear regression of equation (2). Thus, $\Delta m=\Delta a$ is the estimated error on the slope $235 a$ and $\Delta D m$ can be shown to be:

236

(4) $\Delta D m=\left(\left|\frac{1}{m}\right| \times \Delta b+\left|\frac{b}{m^{2}}\right| \times \Delta m\right)$

The errors $\Delta D m$ and $\Delta m$ can then be used to calculate the error on the determination of the dose

$238 D x$. When replicates of a same experiment are used, the mean $\overline{f x}$ is associated to the standard 239 deviation $\sigma f x$ used as an estimation of $\Delta f x$ :

(5) $\Delta D x=\left|\overline{f_{x}} \frac{1}{m}\right| \times \Delta D m+\left|\frac{D m \times \ln \left(\overline{x_{x}}\right)}{m^{2}} \times \overline{f_{x}} \frac{1}{m}\right| \times \Delta m+\left|\frac{D m}{\bar{m}} \times \overline{f_{x}}\left(\frac{1}{m}-1\right)\right| \times \Delta f_{x}$

241 In the present work, $f a$ is the level of DNA photoproducts. In contrast to most works in the field 242 of chromatographic detection of DNA damage, results were not expressed in number of damage 243 per million normal bases but in ratio of mol of damage per mol of DNA bases. The values are 
244 thus very small but range between 0 and 1 as necessary for the use of the MEE. In addition, the $245 f a$ values used in the calculations with the MEE were normalized to the content of bipyrimidine 246 sites at the origin of each photoproducts. For this purpose, we took into account the frequency 247 of the different bipyrimidine dinucleotides in CT-DNA, which are $0.099,0.061,0.053$ and 2480.053 for TT, TC, CT or CC, respectively $(36,37)$.

\section{Polynomial fit}

250 Calculation of the $C I$ was also performed with polynomial fits of the DNA damage data. For 251 this part of the work, results were expressed in number of photoproduct per million normal 252 bases.

253 For Dewars, the experimental data were fitted using a second order polynomial equation. 254 Because no photoproduct is detected in non-exposed DNA, the origin was set to 0. The 255 expression is thus the following:

$256 \quad$ (6) $f a=a \times D^{2}+b \times D$

257 The dose $D x$ necessary for the induction of an effect $f x$ is the positive solution of equation (6):

$258 \quad$ (7) $D x=\frac{-b+\sqrt{b^{2}+4 \times a \times \overline{f x}}}{2 \times a}$

259 with the error of $D x$ being:

260

(8) $\Delta D x=\left|\frac{\overline{f x}}{a \sqrt{b^{2}+4 \times a \times \overline{f x}}}-\frac{\sqrt{b^{2}+4 \times a \times \overline{f x}}}{2 a^{2}}\right| \times \Delta a+\left|\frac{1}{2 a} \times\left(\frac{b}{\sqrt{b^{2}+4 \times a \times \overline{f x}}}-1\right)\right| \times \Delta b+\left|\frac{1}{\sqrt{b^{2}+4 \times a \times \overline{f x}}}\right| \times \Delta f x$

262 Data for CPDs and 64PPs were fitted by a linear regression $f=a \times D$. In this case

263 (9) $D x=\frac{\overline{f x}}{a}$

264 and the error is 
(10) $\Delta D x=\left|\overline{\frac{f x}{a^{2}}}\right| \times \Delta a+\left|\frac{1}{a}\right| \Delta f x$

266

267

268

269

270

271

\section{Kinetic analysis}

Another strategy for the determination of $D x$ involved a precise kinetic analysis. Because the level of damage was low, we considered that the DNA amount remained constant $\left(\mathrm{DNA}_{0}\right)$ and set to $\mathrm{DNA}_{0}=10^{6}$ bases.

\section{CPD}

The formation of CPD in DNA was described as a single first order kinetic reaction.

(11) $C P D=D N A_{0} \times\left(1-e^{-k \times D}\right)$

For the determination of a dose $D x$ leading to a specific level of CPD $(f x)$ :

(12) $D x=-\frac{1}{k} \times \ln \left(1-\frac{f x}{D N A_{0}}\right)$

The error on this value is thus:

(13) $\Delta D x=\left|\frac{1}{k \times(1-\overline{f x})}\right| \times \Delta f x+\left|\frac{\ln \left(1-\frac{\overline{f x}}{D N A_{0}}\right)}{k^{2}}\right| \times \Delta k$ where $\overline{f x}$ is the mean value of the level of CPD in a co-exposure experiment and $\Delta \mathrm{k}$ the estimated error on the rate $\mathrm{k}$.

\section{PP and Dewar}

Formation of 64PP and Dewar results from two consecutive first order reactions DNA $\rightarrow 64 \mathrm{PP} \rightarrow$ Dewar with rate constants $k 1$ and $k 2$ respectively (38).

(14) $64 P P=D N A_{0} \times k 1 \times \frac{e^{-k 1 \times D}-e^{-k 2 \times D}}{k 2-k 1}$

(15) Dewar $=D N A_{0} \times \frac{\left[1-\left(k 2 \times e^{-k 1 \times D}-k 1 \times e^{-k 2 \times D}\right)\right]}{k 2-k 1}$

For the calculation of $k 1$, we considered that all 64PPs produced at a given dose are either in their initial 64PP forms or as Dewars. Therefore, the sum of the levels of 64PP and Dewar can be described, like CPD, by a first order equation with a reaction constant $k 1$. 
(16) $(64 P P+$ Dewar $)=D N A_{0} \times\left(1-e^{-k 1 D}\right)$

287 Log conversion and linear regression yielded the value of $k 1$.

288 For $k 2$, we converted the exponentials in equation (15) by a second order limited development:

289 (17) Dewar $=D N A_{0} \times \frac{k 1 \times k 2 \times D^{2}}{2}$

290 Linear regression of the data with respect to $D^{2}$ provided a fair estimation of $k 2$.

291 The dose $D x$ required for a specific level of Dewar $(f x)$ can be determined from equation (18):

292 (18) $D x=\sqrt{\frac{2 \times \overline{f x}}{D N A_{0} \times k 1 \times k 2}}$

293 The error on this value is thus:

294

(19) $\Delta D x=\frac{\left|\frac{1}{k 1 \times k 2}\right| \times \Delta f x+\left|\frac{-\overline{f x}}{k 2 \times k 1^{2}}\right| \times \Delta k 1+\left|\frac{-\overline{f x}}{k 2^{2} \times k 1}\right| \times \Delta k 2}{D N A_{0} \times \sqrt{\frac{2 \times \overline{f x}}{k 1 \times k 2 \times D N A_{0}}}}$

295 For the determination of $D x$ for 64PPs, we first applied a second order limited development of 296 the exponential functions in equation (14), and obtained equation (20):

297 (20) $64 P P=D N A_{0} \times k 1 \times D-D N A_{0} \times \frac{k 1 \times(k 1+k 2)}{2} \times D^{2}$

298 The dose $D x$ necessary for the induction of a defined level of 64PP (fx) is thus the solution 299 (equation 21) of a second order equation that can be described as the ratio of 2 functions $A$ and $300 \quad B:$

301

(21) $D x=\frac{\left(-k 1 \times D N A_{0}+\sqrt{\left(\left(k 1 \times D N A_{0}\right)^{2}-2 \times \overline{f x} \times\left(k 1^{2}+k 1 \times k 2\right) \times D N A_{0}\right)}\right)}{-\left(k 1^{2}+k 1 \times k 2\right) \times D N A_{0}}=\frac{A}{B}$

302 The other solution of $(20), \frac{\left(-k 1 \times D N A_{0}-\sqrt{\left(\left(k 1 \times D N A_{0}\right)^{2}-2 \times \overline{x x} \times\left(k 1^{2}+k 1 \times k 2\right) \times D N A_{0}\right)}\right)}{-\left(k 1^{2}+k 1 \times k 2\right) \times D N A_{0}}$, provided aberrant values 303 (maximum $D x$ for $f x=0$ and then decreasing $D x$ for increasing $f x$ ). 
305 Calculation of errors requires the derivation of $D x$ with respect to the $k 1, k 2$ and $f x$.

306 Consequently, we calculated $d A$ and $d B$ for each of these 3 parameters:

$\left(22^{\prime}\right) d A(k 1)=-D N A_{0}+\frac{k \& \times D N O_{0}^{e}+\overline{f x} \times D N A_{0} \times(2 k 1+k 2)}{\sqrt{k 1^{2} \times D N A_{0}^{2}-2 \times \overline{f x} \times\left(k 1^{2}+k 1 \times k 2\right) \times D N A_{0}}}$

$\left(22^{\prime \prime}\right) d A(k 2)=\frac{-\overline{f x} \times D N A_{0} \times k 1}{\sqrt{k 1^{2} \times D N A_{0}^{2}-2 \times \overline{f x} \times\left(k 1^{2}+k 1 \times k 2\right) \times D N A_{0}}}$

$\left(22^{\prime \prime \prime}\right) d A(f x)=\frac{-\left(k 1^{2}+k 1 \times k 2\right) \times D N A_{0}}{\sqrt{k 1^{2} \times D N A_{0}^{2}-2 \times \overline{f x} \times\left(k 1^{2}+k 1 \times k 2\right) \times D N A_{0}}}$

$\left(23^{\prime}\right) d B(k 1)=-(2 k 1+k 2) \times D N A_{0}$

$\left(23^{\prime \prime}\right) d B(k 2)=-k 1 \times D N A_{0}$

312 Combination of equations (22') to (23'') provided the error on $D x$ :

(24) $\Delta D x=\left|D x \times\left(\frac{d A(k 1)}{A}-\frac{d B(k 1)}{B}\right)\right| \times \Delta k 1+\left|D x \times\left(\frac{d A(k 2)}{A}-\frac{d B(k 2)}{B}\right)\right| \times \Delta k 2+\left|D x \times \frac{d A(f x)}{A}\right| \times \Delta f x$

\section{Combination index}

315 The Combination Index $C I$ was calculated for each experiment involving combination of UVB

316 and UVA using equation (25):

$$
\text { (25) } C I=\frac{D_{U V B}}{D_{U V B} x}+\frac{D_{U V A}}{D_{U V A} x}
$$

318 with $D_{U V B}$ and $D_{U V A}$ representing the doses of UVB and UVA radiation applied to the sample

319 in the combination experiments, and $D_{U V B} x$ and $D_{U V A} x$ the doses of pure UVB and UVA

320 inducing the same effect $f x$ as the combination $\left(D_{U V B}+D_{U V A}\right) . D_{U V B} x$ and $D_{U V A} x$ were calculated

321 using either equation (3), (7), (9), (12), (18) or (21) depending on the data modeling used and

322 the photoproduct considered.

323 Using the estimated errors $\Delta D x$ calculated for UVB and UVA alone (equations (5), (8), (10),

324 (13), (19) or (24)), and the dose D of UVB and UVA used in the combined exposure, the error on $C I$ was: 
(26) $\Delta C I=\left(D_{U V B} \times \frac{\Delta D U V B x}{D U V B x^{2}}\right)+\left(D_{U V A} \times \frac{\Delta D U V A x}{D U V A x^{2}}\right)$

Effects of UVB and UVA co-exposure shown by the Combination Index and the Median Effect Equation.

331 In a first experiment, we investigated the effect of a combined exposure to UVB and UVA 332 through the classical $C I$ approach with a modeling of the pure UVB and UVA data with the 333 MEE. DNA samples in aqueous solution were exposed to increasing doses of UVB (0.3-12 kJ $\left.334 \mathrm{~m}^{-2}\right)$ or UVA $\left(5-212 \mathrm{~kJ} \mathrm{~m}^{-2}\right)$. In a third experiment, DNA was exposed simultaneously to the 335 same increasing doses of UVB and UVA as those applied alone. The overall UV dose ranged 336 thus between 5.3 and $223 \mathrm{~kJ} \mathrm{~m}^{-2}$. The parameters of the MEE, which are necessary to the 337 determination of the $D x$ values used in the calculation of the $C I$ value, were calculated for UVB 338 for 8 photoproducts: TT CPD, TT 64PP, TT Dewar, TC CPD, TC 64PP, TC Dewar, CT CPD 339 and CC CPD. As previously reported, CC CPD, 64PPs and Dewars were not detected in the 340 samples exposed to UVA with our specific and sensitive HPLC-MS/MS assay (39-41). 341 Therefore, the MEE was determined for UVA only for TT CPD, TC CPD and CT CPD. The fit 342 of UVB data are shown on figure 1. The MEE slightly deviated from the experimental data for 343 CPDs at the largest UVB dose but nicely reproduced the shape of the dose curve formation of 344 64PPs and Dewars. A larger scattering was observed for UVA (data not shown) but the impact 345 of these values on the final $C I$ is very low. 

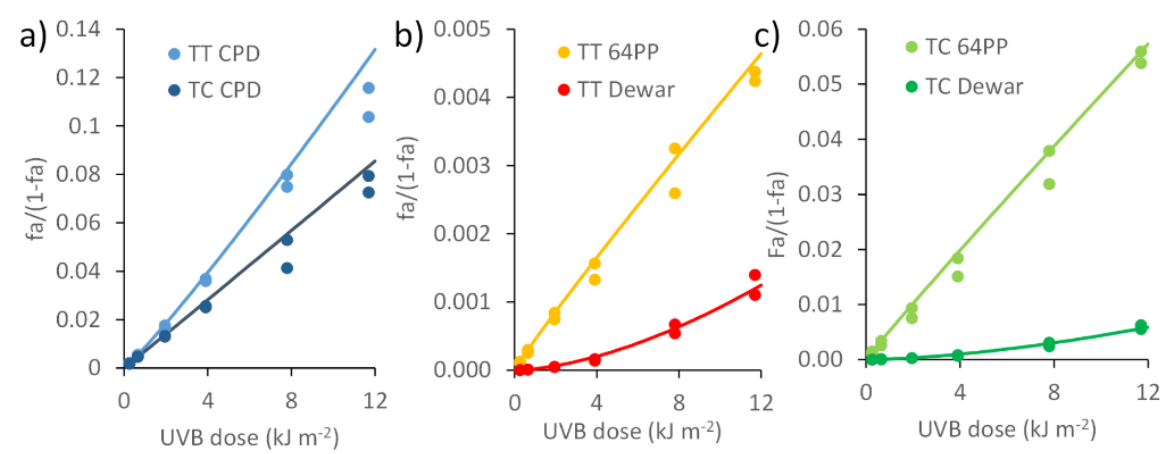

Figure 1: Fit of the dose-dependent formation of pyrimidine dimers in DNA exposed to UVB alone by the median effect equation (MEE) for a) TT and TC CPDs, b) TT 64PP and Dewar c) TC 64PP and Dewar. The marks represent the experimental data while the plain lines are the MEE fits.

These fits of the data were then used to calculate the $C I$ for the different doses of combined UVB+UVA radiation. We were faced with lack of formation of 64PPs and Dewars in the samples exposed to UVA only. This situation is not a priori compatible with the calculation of

$C I$ where the ratio $\frac{D}{D x}$ is taken into account for both factors. To overcome this limitation, we reasoned that a lack of formation could be seen as the necessity to apply an infinite dose to induce an effect. Consequently, the $D x$ value in the ratio involved in the calculation of $C I$ is infinite and the ratio is null. We thus did not consider the term $\frac{D_{U V A}}{D_{U V A x}}$ in the calculation of $C I$ for 64PPs, Dewars and CC CPD. Another improvement of the $C I$ formalism in the present study was the accurate estimation of the error of the value of $C I$. A possible approach could be a replication of the whole experiment and the calculation of the mean and the variance. However, such an approach is not satisfactory because it does not really take into account the error on the

362 MEE parameters and mostly reflects the variation in the results of the combination experiment.

363 Therefore, we rather took into account the propagation of the estimated errors of the fitted 364 parameters of the linear regression aimed at calculating the $m$ and $D m$ coefficients of the MEE.

365 A first observation regarding the calculated $C I$ in this experiment was that the values were similar at all doses and close to 1 for the four studied CPDs (Fig. 2). This reflects that, in the 
UVB and UVA ranges applied, formation of CPDs is a simple first order reaction. The 368 photoreversion observed with UVC radiation $(42,43)$, which is explained by a residual absorption by CPD of these high-energy photons, does not take place. The present results also confirmed that UVA induces the formation of T-containing CPDs but in much lower yield than UVB. The observed ratio here was approximately 2000 for TT CPD. Consequently, additivity 372 is observed for CPD in case of co-exposure to UVB and UVA and the $C I$ value is 1.

373

374

375 376

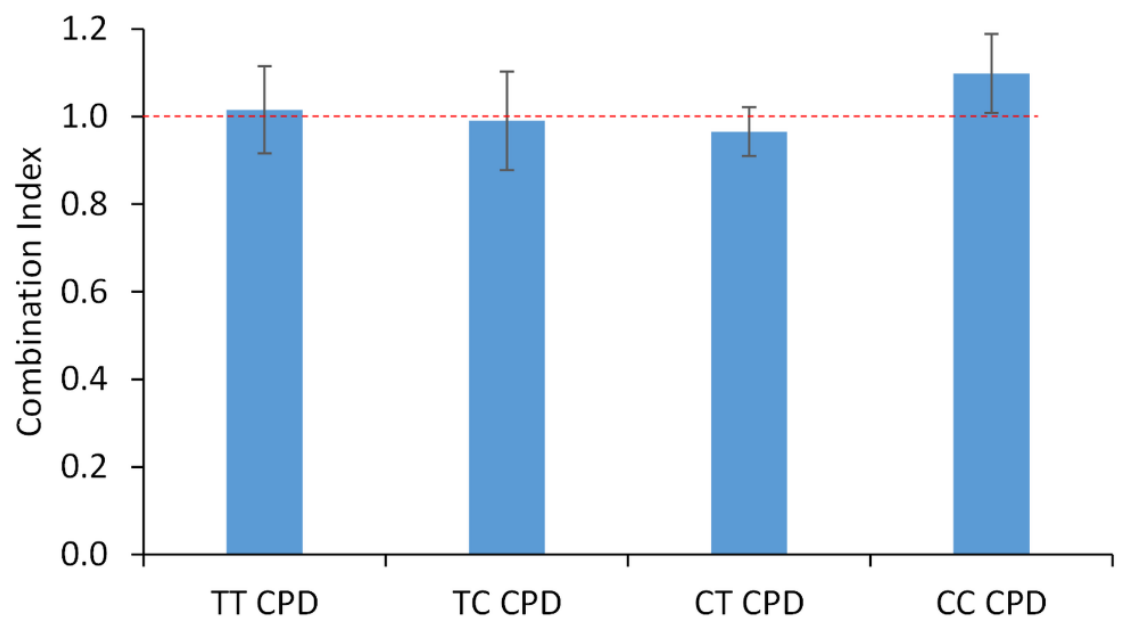

Figure 2: CI calculated for CPD upon exposure of DNA to UVB and UVA in a 1:18 ratio. The reported values are the means ( \pm standard deviation) of results obtained in duplicate at six different $U V$ doses.

In contrast to the formation of CPDs, the formation of 64PPs and Dewars was greatly affected by the presence of UVA during UVB irradiation. 64PPs are efficiently produced by UVB but not UVA. Both UVB and UVA are absorbed by the pyrimidone ring of 64PPs and convert them into Dewars. In isolated 64PPs like photoproducts of dinucleoside monophosphates, this reaction exhibits a maximal efficiency in the UVB range as 64PPs have a maximal absorption at approximately $320 \mathrm{~nm}(31,19,32,33)$. However, UVA is more efficient than UVB at photoisomerizing 64PPs in DNA because of the shielding effects of the overwhelming amount of the normal bases in the latter wavelengths range. This is observed in isolated as well as in cellular DNA (34) and could be modeled based on available absorption features and quantum 
yield data (18). The calculated CIs for the TT and TC 64PPs and Dewars unambiguously reflect

387 this chain of reactions (Table 1). The value of the $C I$ is below 1 for Dewars. The mean value was 0.3 and 0.4 for TT Dewar and TC Dewar, respectively, and thus corresponds to a synergy effect in the combined exposure, namely a favored formation in the presence of UVA. The opposite trend is observed for 64PPs since their $C I$ are larger than 1 . The $C I$ analysis even reflects known differences between the photochemistry of TC and TT 64PPs. The antagonism of the formation of 64PP by UVA and the synergistic formation of Dewar are stronger for the TT than the TC photoproducts. Actually, it was observed, from isolated DNA (44) to mammalian cells $(45,46)$ and marine microorganisms collected on the field $(47)$, that the yield of conversion into Dewars was larger for TT 64PP than TC 64PP. The quantum yield for the two photoisomerization reactions are similar with values of $2.010^{-4}$ and $1.810^{-4}$, respectively (33). The absorption coefficient of TC 64PP is 3 times lower than that of TT 64PP (48), which partly explains the difference in the yield of photoisomerization. A maximum absorption is observed at $325 \mathrm{~nm}$ for TT 64PP (19) and $315 \mathrm{~nm}$ (32) for TC 64PP. Therefore, the lower absorption coefficient of TC 64PP is compensated by the emission of the UVB source used in 401 this work that exhibits a maximum at $312 \mathrm{~nm}$. More interesting for the topic of our work is that 402 calculations based on known absorption coefficients and quantum yields provided evidence that the photoisomerization yield of TC 64PP is still 3-fold lower than that of TT 64PP in combination of 300 and either 315,320 or $340 \mathrm{~nm}$ light (34). This larger synergy in the isomerization of TT 64PP compared to TC 66PP is reflected by the lower value of CI for TT

406 Dewar than TC Dewar. Another observation is the increase in the value of the CI of 64PPs 407 when the applied dose increased. This feature may be explained by the fact that, upon exposure 408 to combination of UVB and UVA, the level of 64PPs tends to a plateau while the increase is 409 more monotonous with pure UVB. Consequently, the antagonist effect is more pronounced 410 when the dose increases. Regarding the error on the determination of the $C I$, a general trend 
411 observed for all photoproducts is that it is larger at the lowest doses. This can be explained by

412 a less accurate determination of the amount of photoproducts or a less reproducible applied UV

413 dose. In addition, the weight of the low values at the lowest dose is lower than that at high dose

414 in the regression providing the parameters of the MEE. It is thus expected that the accuracy is

415 lower at low dose.

416 Table 1: Values of the combination index (CI) determined for increasing doses of UVA and $417 U V B$ applied in a 18:1 ratio. The reported total $U V$ dose is expressed in $\mathrm{kJ} \mathrm{m}^{-2}$. The errors were 418 obtained as described in the calculation section and included errors on the parameters of the 419 MEE and the Dx dose.

\begin{tabular}{|c|c|c|c|c|c|c|}
\hline \multicolumn{7}{|c}{ Cl calculated by the Median Effect Equation } \\
\hline UV dose & TT 64PP & TT Dewar & TC 64PP & TC Dewar & TT CPD & TC CPD \\
\hline 5 & $1.12 \pm 0.63$ & $0.32 \pm 0.11$ & $1.23 \pm 0.75$ & $0.44 \pm 0.20$ & $0.83 \pm 0.12$ & $0.83 \pm 0.23$ \\
\hline 12 & $1.27 \pm 0.57$ & $0.31 \pm 0.12$ & $1.28 \pm 0.67$ & $0.48 \pm 0.22$ & $0.99 \pm 0.18$ & $0.87 \pm 0.23$ \\
\hline 37 & $1.53 \pm 0.71$ & $0.29 \pm 0.08$ & $1.33 \pm 0.62$ & $0.45 \pm 0.19$ & $1.13 \pm 0.21$ & $1.05 \pm 0.29$ \\
\hline 75 & $2.04 \pm 0.96$ & $0.28 \pm 0.08$ & $1.30 \pm 0.73$ & $0.38 \pm 0.16$ & $1.07 \pm 0.15$ & $1.03 \pm 0.26$ \\
\hline 149 & $3.30 \pm 1.65$ & $0.30 \pm 0.08$ & $1.44 \pm 0.74$ & $0.34 \pm 0.13$ & $1.03 \pm 0.16$ & $1.09 \pm 0.44$ \\
\hline 223 & $4.75 \pm 2.34$ & $0.33 \pm 0.08$ & $1.69 \pm 0.82$ & $0.32 \pm 0.10$ & $1.04 \pm 0.19$ & $1.07 \pm 0.30$ \\
\hline
\end{tabular}

421 The results clearly show that calculation of the $C I$ based on the MEE as defined by Chou (25) 422 is well suited for establishing additive or non-additive effects in photochemically-induced 423 processes like formation of DNA damage. CI nicely reflects the additivity between UVB and 424 UVA in the induction of CPDs, the lower levels of 64PPs in the presence of UVA and the larger 425 amounts of Dewars upon co-exposure to UVB and UVA. The present work illustrates the ease 426 of the procedure that requires only three experiments with a few doses to obtain an indication 427 on the occurrence of synergistic or antagonist effects over a wide range of doses. In the present 428 work, we could explore a dose range corresponding to a factor of 45 between the lowest and 429 the largest dose with only 6 points for UVB, UVA and UVB+UVA. 


\section{Calculation of $C I$ with other fitting functions}

431 The results presented above for UVB and UVA is an illustration, in addition to countless 432 examples with drugs and chemical, of the power of the $C I$ approach to identify synergism and 433 antagonism in mixtures. The definition of this parameter was inferred from extensive theoretical 434 work based on the mass action law and the concept of the MEE. Yet, the MEE was designed 435 for drug-target interactions and cannot reflect all possible biological responses. This is the case 436 for all the cellular processes that do not exhibit a dose-response shape with a plateau and that 437 cannot be normalized between 0 and 1 . An important example is that of gene expression that 438 can be larger than one when increased with respect to a control sample and that do not have a 439 theoretical upper limit. We reasoned that in these cases, the $C I$ can still be calculated because 440 it only depends on ratio of doses. We thus explored whether the role of the MEE in the initial 441 strategy developed by Chou (25) could be played by other mathematical functions. This would 442 allow fitting any shape of dose-dependent cellular responses. In addition, the absolute 443 requirement of responses ranging between 0 and 1 permitting the MEE to be applied can be 444 avoided by using other equations. The limiting ingredient to the determination of $C I$ is that the 445 fitted function for the effect with respect to the dose may easily be inverted to determine $D x$ for 446 the observed effect $f x$ of mixtures. It is yet important to limit the number of fitting parameters 447 in order to avoid overfitting the experimental data of the pure dose effects.

448 We thus used the set of experimental results analyzed above with the MEE equation with other 449 functions aimed at fitting the data for pure UVA and UVB and calculating Dx from fx. We first 450 applied polynomial regressions. Linear regressions were used for CPDs and 64PPs, and second 451 order polynomial regressions for Dewars. In a first attempt, second order polynomial 452 regressions were also used for 64PPs but errors were very large and the obtained CIs close to 453 those obtained with linear regression (data not shown). The $C I$ values obtained for the four 454 CPDs, and for TT and TC 64PPs and Dewars were very similar whether a polynomic regression 
455 (Table 2) or the MEE were used (Table 1). The mean ratio between values provided by the 456 MEE and the polynomial regression is 1.06 . This result shows that the $C I$ can be used with any 457 fitting function, even if it is not based on known physical-chemical processes like MEE that is 458 based on the mass action law. In addition, the values of the level of photoproducts used in the 459 polynomial regressions were expressed as number of photoproducts per million bases and were 460 thus larger than 1 (up to 9000 per $10^{6}$ bases for TT CPD).

461 Table 2: Values of the combination index (CI) determined for increasing doses of UVA and 462 UVB applied in an 18:1 ratio, with the use of data fitting other than the MEE. The reported total UV dose is expressed in $\mathrm{kJ} \mathrm{m}^{-2}$. The errors were obtained as described in the calculation section and included errors on the parameters of the polynomial regression or kinetic equations, of the Dx dose and on the final calculation of CI.

\begin{tabular}{|c|c|c|c|c|c|c|}
\hline \multicolumn{7}{|c|}{ Cl calculated by linear or second order polynomial regression } \\
\hline UV dose & TT 64PP & TT Dewar & TC 64PP & TC Dewar & TT CPD & TC CPD \\
\hline 5 & $0.85 \pm 0.14$ & $0.28 \pm 0.04$ & $0.96 \pm 0.24$ & $0.41 \pm 0.27$ & $0.92 \pm 0.14$ & $0.71 \pm 0.20$ \\
\hline 12 & $1.01 \pm 0.06$ & $0.27 \pm 0.03$ & $1.05 \pm 0.18$ & $0.41 \pm 0.17$ & $1.06 \pm 0.15$ & $0.76 \pm 0.12$ \\
\hline 37 & $1.29 \pm 0.13$ & $0.27 \pm 0.01$ & $1.17 \pm 0.15$ & $0.40 \pm 0.08$ & $1.16 \pm 0.10$ & $0.94 \pm 0.06$ \\
\hline 75 & $1.75 \pm 0.20$ & $0.30 \pm 0.02$ & $1.19 \pm 0.28$ & $0.38 \pm 0.06$ & $1.06 \pm 0.18$ & $0.95 \pm 0.02$ \\
\hline 149 & $2.87 \pm 0.42$ & $0.35 \pm 0.02$ & $1.37 \pm 0.28$ & $0.39 \pm 0.04$ & $1.00 \pm 0.13$ & $1.02 \pm 0.03$ \\
\hline 224 & $4.15 \pm 0.57$ & $0.40 \pm 0.01$ & $1.64 \pm 0.30$ & $0.40 \pm 0.02$ & $0.99 \pm 0.06$ & $1.02 \pm 0.13$ \\
\hline \multicolumn{7}{|c|}{ Cl calculated by kinetic analysis } \\
\hline UV dose & TT $64 \mathrm{PP}$ & TT Dewar & TC $64 \mathrm{PP}$ & TC Dewar & TT CPD & TC CPD \\
\hline 5 & $1.07 \pm 0.30$ & $0.21 \pm 0.01$ & $1.07 \pm 0.35$ & $0.25 \pm 0.01$ & $0.92 \pm 0.14$ & $0.71 \pm 0.21$ \\
\hline 12 & $1.27 \pm 0.23$ & $0.24 \pm 0.02$ & $1.17 \pm 0.29$ & $0.32 \pm 0.02$ & $1.06 \pm 0.15$ & $0.76 \pm 0.12$ \\
\hline 37 & $1.60 \pm 0.35$ & $0.27 \pm 0.01$ & $1.29 \pm 0.27$ & $0.38 \pm 0.02$ & $1.16 \pm 0.11$ & $0.94 \pm 0.06$ \\
\hline 75 & $2.15 \pm 0.51$ & $0.30 \pm 0.02$ & $1.30 \pm 0.41$ & $0.38 \pm 0.03$ & $1.07 \pm 0.18$ & $0.95 \pm 0.03$ \\
\hline 149 & $3.49 \pm 0.95$ & $0.35 \pm 0.02$ & $1.46 \pm 0.43$ & $0.40 \pm 0.02$ & $1.00 \pm 0.13$ & $1.02 \pm 0.03$ \\
\hline 224 & $5.03 \pm 1.33$ & $0.41 \pm 0.02$ & $1.71 \pm 0.47$ & $0.42 \pm 0.01$ & $0.99 \pm 0.06$ & $1.02 \pm 0.13$ \\
\hline
\end{tabular}

466

467 Because the photochemical reactions leading to the formation of the three types of 468 photoproducts are well known, we could also apply a more rigorous approach for the fit of the 469 experimental data by using chemical kinetic equations. Formation of CPDs was described as a 470 simple first order reaction characterized by a reaction constant $k$. In contrast, formation of 64PPs 471 and Dewars were described as two consecutive reactions with reaction constants $k 1$ and $k 2$. The 
constant $k 1$ was related to the initial formation of 64PPs and second $k 2$ reflected their conversion into Dewar. As described in the "Data modeling" part, these equations were used to 474 fit the data and calculate the $D x$ values necessary for the determination of the $C I$. Like for polynomial regression, the obtained $C I$ values (Table 2) were very close to those obtained using 476 the MEE (Table 1). The mean ratio between values provided by the MEE and the kinetic 477 analysis is 1.07 . Obviously, this type of kinetic data analysis is only possible when the basic 478 steps of a mechanisms are well identified. In this case, the use of a strategy like the $C I$ is thus not necessary to predict occurrence of non-additivity. However, the fact that similar results were obtained for the $C I$ with various fitting strategies like MEE or polynomial regressions on the one hand, and more realistic calculations based on kinetic parameters on the other, validates 482 the two former approaches. Interestingly, the kinetic analysis emphasized a limitation to the $C I$ 483 determination that concerns all fitting functions. The dose dependent-formation of 64PPs exhibits a bell-shaped curve. Calculation of the theoretical level of TT 64PP for large doses based on equation (15) shows that a maximal level is reached at approximately $200 \mathrm{~kJ} \mathrm{~m}^{-2}$. At this dose, the level of photoproduct is 1180 TT 64PP per million bases. Consequently, above 487 this dose, the calculation of $D x$ will provide 2 relevant values for a mixture effect $f x$ and it will 488 not be possible to calculate a $C I$. This was not the case in our experiments where the dose was lower than $12 \mathrm{~kJ} \mathrm{~m}^{-2} \mathrm{UVB}$. This example of the use of the kinetic equations illustrates that the use of functions other than the MEE for the determination of $C I$ has to be carried out at doses

491 below maximal responses for bell-shaped curves.

492 Interesting observations can also be made regarding the errors on the $C I$. When the latter 493 parameter was calculated on the basis of the MEE, the lowest relative errors were obtained for 494 Dewars, with values of approximately $30 \%$ at the largest dose. However, for both polynomial 495 regression and kinetic analysis, this error is on the average 5 times lower than with the MEE. A 496 similar trend was observed for 64PPs when a linear regression was used for the determination 
of the $C I$ compared to MEE. The relative errors on $C I$ are $12 \%$ and $48 \%$, respectively, for TT 64PP at the largest dose. The corresponding values are 19 and $50 \%$ for TC 64PP. Comparison of errors was also in favor of the kinetic analysis versus MEE, although to a lesser extent than the polynomial regressions. With the kinetic analysis, the relative errors on the $C I$ for TT 64PP and TC 64PP at the largest dose were 25 and $27 \%$, respectively. From the back propagation equations, it is possible to estimate the relative impact of the experimental data and the fitting parameters on the error on $D x(\Delta D x)$ and consequently on the $C I$ error $(\Delta C I)$. If $\Delta D x$ is dominated by the parameters of the fits, other fitting functions may require to be considered or experiments of pure factors with a larger dose range. On the contrary, if $\Delta D x$ is dominated by experimental data on mixture effects, new experiments of mixtures with more replicates may be required. For TT 64PP at the largest dose, $\Delta D x$ determined with the MEE originates in $42 \%$, $32 \%$ and $26 \%$ from error on $D m, m$ and $f x$, respectively. In this case, improvement of the accuracy of the fit seems necessary. For the kinetic analysis approach applied to TT 64PP, the proportions of $\Delta D x$ originating from $k 1, k 2$ and $f x$ were $22 \%, 31 \%$ and $47 \%$ at the largest dose. The result show a strong contribution of the fit. For the second order polynomial fit of TT 64PP (not discussed in this work), $\Delta C I$ was 2000 for a $C I$ of 4.5 and the error on the quadratic coefficient represented $99.9 \%$ of $\Delta D x$. With the linear regression, a much lower error was obtained (0.57). The contribution of the errors to $\Delta D x$ were 14 and $86 \%$ for the value of the slope and $f x$, respectively. In this case, increasing the quality of the experimental results of mixtures, for example by using more replicates, could improve the estimation of $C I$. The second order polynomial fit was found to be very accurate for TT Dewar with an error of $4 \%$ on $C I$ at the largest dose. In this case the contribution of the errors to $\Delta \mathrm{Dx}$ were $40 \%, 56 \%$ and $4 \%$ for quadratic term, the linear term and $f x$, respectively. These observations point again to a significant contribution of the quality of the fit to the error on the $C I$. Altogether, these results suggests that $\Delta \mathrm{Dx}$ and $\Delta C I$ are mostly explained by errors on the fit, especially when the overall 
errors are large. This shows that the choice of the fitting function is a key parameter in the accuracy of the determination of the $C I$. In the average, the MEE predicts $C I$ with an error on the average 4 times larger than polynomial regressions or kinetic analysis, while the $C I$ values are similar with all approaches.

\section{Dependence of $C I$ on the irradiation conditions}

We then performed a series of experiments still involving a combination of UVA and UVB in order to determine whether the $C I$ was sensitive to the exposure protocols. In this experiment and in all the rest of the work, we relied on the initial strategy designed by Chou et al. $(25,27)$ for the calculation of the CI with the MEE. We first investigated whether the $C I$ varied according to the UVA/UVB ratio. We then studied the impact of the order in which UVA was combined with UVB, either simultaneously or one after the other. For these experiments, only one sample was collected at each dose. Consequently, we did not calculate errors.

\section{Evolution of the CI at different $U V A$ to $U V B$ ratios}

In the previous study, UVA and UVB were applied at a constant ratio of 18:1. Yet, it is anticipated that the efficiency of conversion of 64PPs into Dewars depends on the relative intensities of UVB and UVA. Therefore, we calculated the $C I$ through a MEE fitting for different exposure conditions exhibiting increasing UVA to UVB ratios. For this purpose, we kept the UVA fluence constant and decreased that of the UVB source. The results unambiguously showed a decrease of the value of the $C I$ related to Dewars with increasing ratio, namely an increase in the synergistic effect, when the UVA to UVB ratio increased (Fig. 3). Conversely, the $C I$ for TT and TC 64PPs was larger than 1 under all exposure conditions, showing the antagonist effect of UVA. The effect was not as strong as those shown on Figure 1 because the total UV doses applied were lower. Like in our experiments at constant 
UVA/UVB ratio, the values of the $C I$ for TC Dewar were larger than those for the TT Dewar.

547 The mean ratio between the $C I$ of TC Dewar and TT Dewar at different applied doses ranged 548 from 1.2 for a UVA/UVB ratio of 3.3, to 1.9 for a UVA/UVB ratio of 21.8. These observations 549 reflect the more efficient isomerization of TT 64PP in the UVA range compared to TC 64PP. 550 This experiment illustrates that the $C I$ is more than a semi-quantitative indication of the 551 non-additivity of response in co-exposures. The modulation of DNA photochemistry induced 552 by changing the ratio between the fluence of UVB and UVA by a value of 2 or 3 was enough 553 to be reflected in the values of the $C I$.
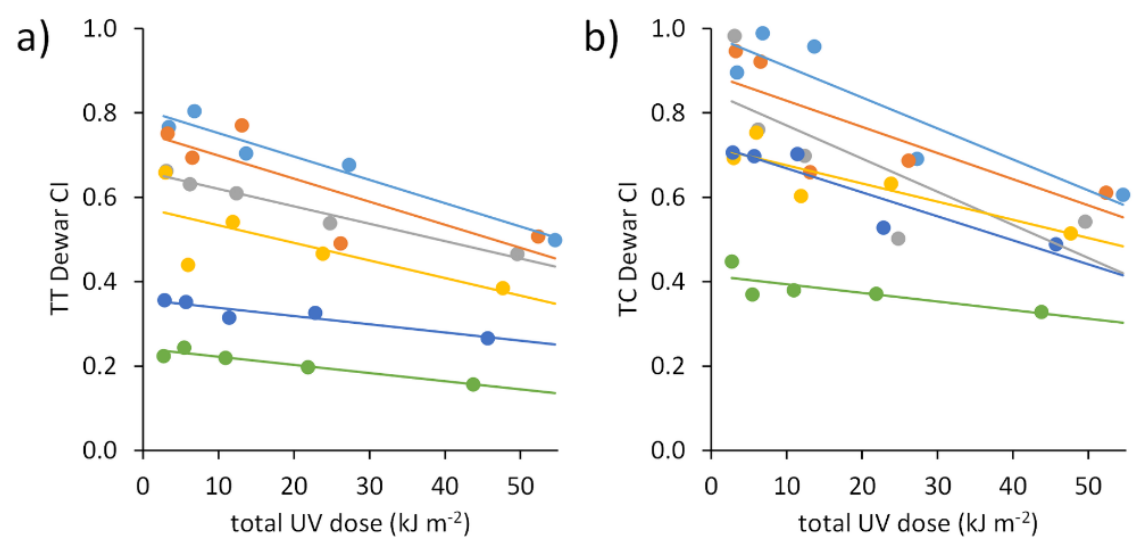

UVA/UVB ratio: $\bullet 3.3 \cdot 4.0 \cdot 5.4 \cdot 7.2 \cdot 10.9 \cdot 21.8$

Figure 3: Value of the CI for a) TT Dewar and b) TC Dewar in DNA samples exposed to radiation with various $U V A / U V B$ ratios.

561 In the subsequent study, we compared the results of UVB and UVA co-exposure experiments 562 where the two radiation ranges were applied either simultaneously or one after the other. 563 Experiments involving pure UVA and UVB were also performed in order to calculate the $C I$. 564 As observed in all the other experiments, the $C I$ values determined for CPDs were close to 1 , 
irrespectively of the irradiation conditions and of the dose (data not shown). Interestingly, the

566 total dose of UVA and UVB received by the DNA samples was the same in the UVA $\rightarrow$ UVB,

$567 \mathrm{UVB}+\mathrm{UVA}$ and UVB $\rightarrow$ UVA protocols. Yet, differences were observed in the

568 photoisomerization yield. In the first case (UVA $\rightarrow$ UVB), the efficiency is not different than

569 with UVB alone as shown by the $C I$ close to 1 for 64PPs and Dewars. This is expected since

570 UVA is applied first and cannot be absorbed by 64PPs produced by UVB. This was not the case

571 for the two other irradiation protocols. For TT 64PP, the value of the $C I$ increased continuously

572 from 0.98 to 1.30 with increasing dose for UVB+UVA and from 1.26 to 1.69 for UVB $\rightarrow$ UVA.

573 The same trend was observed for TC 64PP. The CI values obtained at the lowest and largest

574 UV doses were 1.00 to 1.14 with increasing dose for UVB+UVA and from 1.04 to 1.29 for

575 UVB $\rightarrow$ UVA. A mirror effect was observed on the Dewar data. As shown on figure 4, the $C I$

576 value for both TT and TC Dewars were 1 on the average in the UVA $\rightarrow$ UVB protocol, but lower

577 in the UVB+UVA and UVB $\rightarrow$ UVA experiments. The mean values are 0.44 and 0.28 for TT

578 Dewar for UVB+UVA and UVB $\rightarrow$ UVA, respectively. The corresponding values for TC Dewar

579 are 0.74 and 0.56 . (Fig. 4). In summary, the $C I$ for 64PPs was larger for UVB $\rightarrow$ UVA than

580 UVB+UVA while the opposite trend was observed for Dewars. Interestingly, the $C I$ of the TT

581 64PP is more dose-dependent than that of TC 64PP. This can be explained by the fact that the

582 photoisomerization reaction is more efficient. Consequently, the trend to a plateau for 64PP and

583 the quadratic shape of the formation of Dewar are more pronounced for TT than TC. In the

584 calculation of the $C I$, this is reflected by a larger dose dependence for TT than TC 585 photoproducts. 

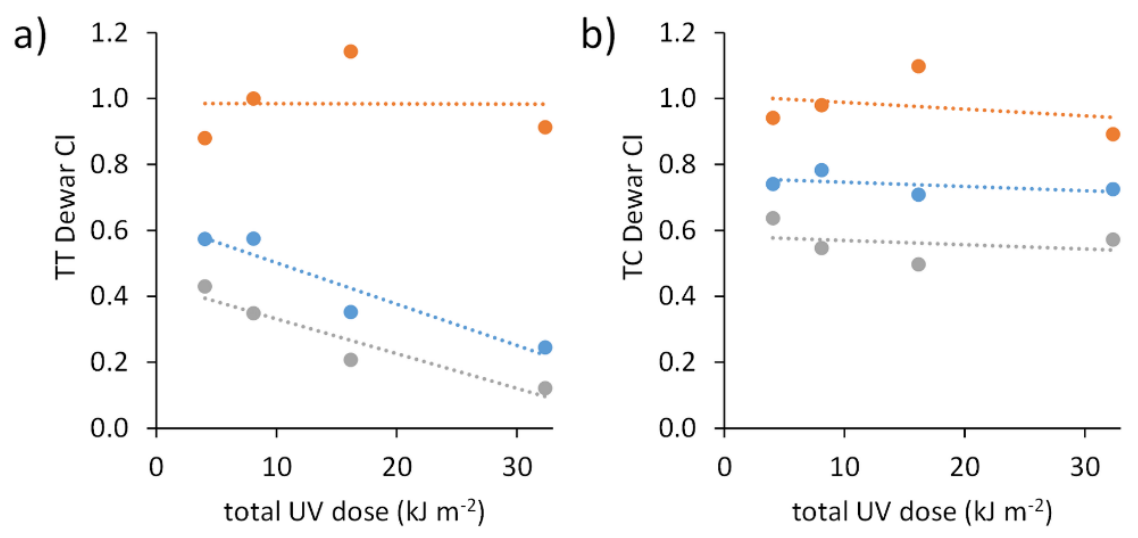

Irradiation condition: $\bullet U V B+U V A \cdot U V A \rightarrow U V B \cdot U V B \rightarrow U V A$

Figure 4: variation of CI of TT and TC Dewars for different irradiation protocols. UVB and $U V A$ were applied simultaneously (UVB+UVA), or successively $U V B \rightarrow U V A$ or $U V A \rightarrow U V B$.

591 The bulk of these observations unambiguously shows that the photoisomerization is more

592 efficient with UVB $\rightarrow$ UVA than UVB+UVA. This result can be explained by the fact that when

593 UVA is applied after UVB, all UVB-induced 64PPs absorb the maximal amount of UVA. When

594 both wavelength ranges are applied simultaneously, the same amount of 64PPs as in the 595 UVB $\rightarrow$ UVA design are produced. However, those formed close to the end of irradiation are exposed to a lower dose of UVA than those produced at the beginning. Consequently, their

597 yield of photoisomerization is lower. A more comprehensive explanation can be found in figure 5985 that shows, for the 40 min experiments, the evolution of the level of TT 64PP and TT Dewar 599 under the different exposure protocols. The x-scale of the graph is in min because total UV 600 doses are quite different for UVB alone and combination of UVB and UVA. CI reported in 601 figure 4 were the final values for each irradiation time $(5+5,10+10,20+20$ or $40+40 \mathrm{~min})$. 
602

603

604

605

606

607

608

609

610

611

612

613

\section{Combination of 3 UV sources}

615 The results presented above involved only a UVB and a UVA source. Yet, photobiological

616 processes could be modulated by combinations of more than 2 ranges of wavelengths. We took advantage that the $C I$ can be used with more than two factors to explore a ternary combination

Figure 5: Formation of a) TT 64PP and b) TT Dewar in isolated DNA exposed to either UVB alone or to UVB and UVA in different combinations. UVB+UVA: simultaneous exposure; $U V B \rightarrow U V A: U V B$ alone followed by UVA alone; UVA $\rightarrow U V B: U V A$ alone followed by UVB alone. The graphs were obtained by using equations (14) and (15). The value of $k 1$ was obtained in the UVB experiment. A value $k 2_{U V B}$ was determined for pure UVB as explained in the calculation part. A similar approach was applied for the determination of $k 2_{U V B+U V A}$ in the $U V B+U V A$ experiment. The difference between these 2 values yielded $k_{U V A}$ used for the fit of the UVB $\rightarrow U V A$ data. The values of $k 1, k 2_{U V B}, k 2_{U V B+U V A}$ and $k 2_{U V A}$ were found to be $410^{-7}$, $0.002,0.010$ and $0.008 \mathrm{~min}^{-1}$, respectively. 
$619 \operatorname{UVB}(310 \pm 5 \mathrm{~nm}), \mathrm{UVA} 2(340 \pm 13 \mathrm{~nm})$ and UVA1 $(386 \pm 13.5 \mathrm{~nm})$. In addition to each radiation

620 alone and to the ternary combination, we exposed solutions of isolated DNA to a combination 621 of UVB and UVA2 $(310+340 \mathrm{~nm})$ and a combination of UVB and UVA1 $(310+386 \mathrm{~nm})$. As 622 observed with the full UVA spectrum, neither UVA1 nor UVA2 led to the formation of 64PPs 623 and Dewars. We observed that the $C I$ was 1 for 64PPs and Dewars for the $310+386 \mathrm{~nm}$ 624 combination (Fig. 6), suggesting that UVA1 is not efficient in the photoisomerization of 64PP. 625 In contrast, the $C I$ for Dewars in the $310+340 \mathrm{~nm}$ experiments were below 0.5 and statistically 626 significantly lower than those observed for $310+386 \mathrm{~nm}$. The same observations were made in 627 the ternary combination, showing that addition of UVA1 to the UVB/UVA2 radiation did not 628 increase the photoisomerization yield of 64PPs. It should be noticed that a statistically 629 significant larger $C I$ was obtained for TC $64 \mathrm{PP}$ in the $310+340 \mathrm{~nm}$ irradiation compared to $630310+386$, as well as between the $310+386$ and the $310+340+386$ experiments. The latter 631 difference was also statistically significant for TT 64PP, and the comparison between $310+340$ 632 and 310+386 exhibited the same trend of a CI closer to 1 under the latter condition. These 633 results strongly suggest an antagonist effect of UVA2, but not of UVA1, on the formation of 634 64PPs. Combined with observation of synergistic effects of UVA2 but not UVA1 on the 635 formation of Dewars, the $C I$ analysis allowed us to conclude that UVA1 does not participate to 636 the photoisomerization of 64PPs, in agreement with their known absorption properties. 


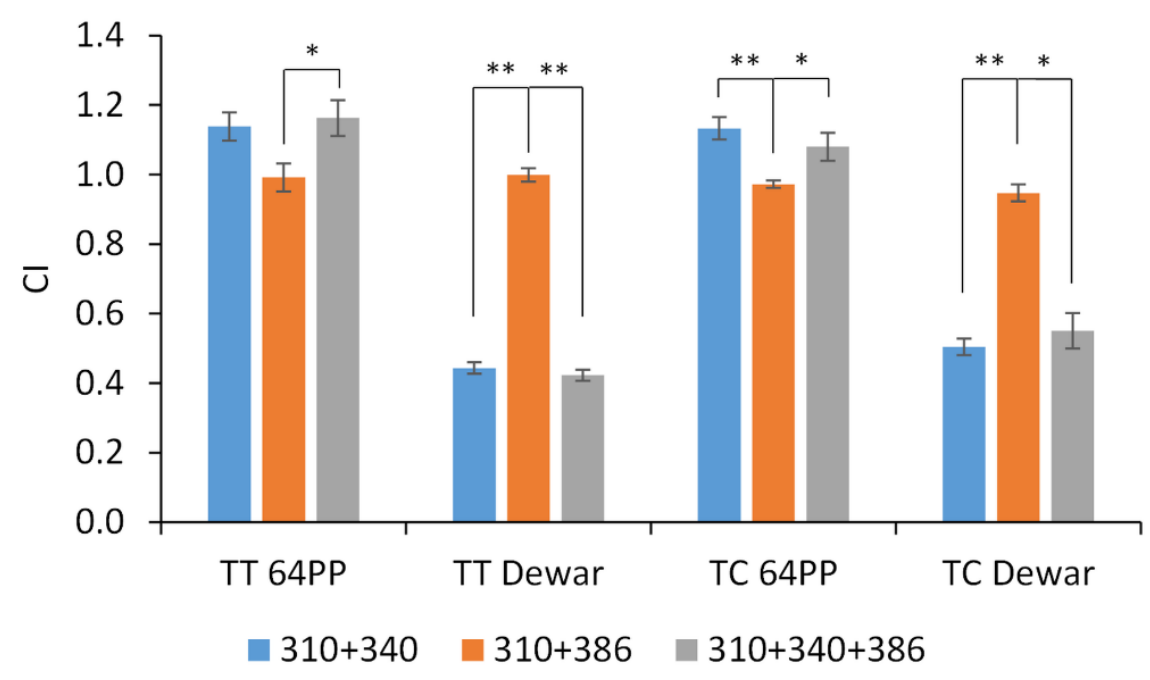

637

638

639

640

641

642

643

644

645

646

647

648

649

650

651

652

653

654

Figure 6: CI determined for TT and TC 64PPs and Dewars in DNA samples exposed to combination of UVB, UVB and UVA1 and UVB and UVA2. The reported results are means \pm SEM determined in triplicate at 4 different total $U V$ doses $(n=12)$. The statistical significance of the differences, evaluated by a one-way ANOVA, were $p<0.05$ (*) or $p<0.01$ (**).

\section{CONCLUSION}

In the present work, we proposed the use of the combination index as a tool to identify non additive effects upon co-exposure to different radiation ranges. Application of this approach to the well-known photochemistry of DNA shows that the $C I$ strategy is as efficient with light as it is with chemicals. We also provided some improvements to the original design by showing that fitting functions other than the MEE can be used. In particular, we show that regressions that are not necessarily based on physico-chemical principles like the mass action law for MEE or chemical kinetics analysis are well suited. In our case, linear and second order polynomial fits of the data provided $C I$ values similar to those obtained with the MEE or kinetic analysis, with the lowest incertitude. In addition, these fitting functions can exhibit any dose-response curves, except bell-shaped responses. Another advantage of the use of such fitting functions is that the value of the considered effect does not have to be lower than 1 as in the MEE, which 
would be a strong limitation for example for the induction of all photobiological effects normalized to a control like gene expression or protein synthesis. Further developments will involve application of the combination index strategy to investigations of the effect of complex exposures on living systems with biologically relevant endpoints. Another long term project would be to incorporate the non-additivity brought by $C I$ methodology in a generalized action spectra for the estimation of dose effects with complex light sources like solar spectra.

\section{ACKNOWLEDGMENTS}

This work was supported by the Labex ARCANE and the CBH-EUR-GS (ANR-17-EURE-0003).

\section{REFERENCES}

1. Björn, L. O. (2015) Action spectroscopy in biology. In Photobiology: The Science of Light and Life. (Edited by L. O. Björn), pp. 85-96. Springer Science + Business Media, NewYork.

2. Sliney, D. H. (2006) Photobiological measurements and obtaining action spectra. In Cell Biology and Instrumentation: UV Radiation, Nitric Oxide, and Cell Death in Plants (Edited by Y. Blume, D. J. Durzan and P. Smertenko), pp. 11-26. IOS Press, Amsterdam.

3. Setlow, R. (1957) Action spectroscopy. Adv. Biol. Medic. Phys. 5, 37-74.

4. Schäfer, E. and L. Fukshansky (1984) Action spectroscopy. In Techniques in Photomorphogenesis. (Edited by H. Smith and M. G. Holmes). Academic Press, NewYork.

5. Withrow, R. B., W. H. Klein and V. Elstad (1957) Action Spectra of Photomorphogenic Induction and Its Photoinactivation. Plant Physiol. 32, 453-462.

6. Essen, L. O. and T. Klar (2006) Light-driven DNA repair by photolyases. Cell. Mol. Life Sci. 63, 1266-1277.

7. Weber, S. (2005) Light-driven enzymatic catalysis of DNA repair: a review of recent biophysical studies on photolyase. Biochim. Biophys. Acta 1707, 1-23. 
8. Douki, T. (2021) Wavelengths and temporal effects on the response of mammalian cells to UV radiation: Limitations of action spectra illustrated by genotoxicity. $J$ Photochem Photobiol B 217, 112169.

9. Damian, D. L., Y. J. Matthews, T. A. Phan and G. M. Halliday (2011) An action spectrum for ultraviolet radiation-induced immunosuppression in humans. Br. J. Dermatol. 164, 657-659.

10. Garssen, J., F. de Gruijl, D. Mol, A. de Klerk, P. Roholl and H. Van Loveren (2001) UVA exposure affects UVB and cis-urocanic acid-induced systemic suppression of immune responses in Listeria monocytogenes-infected balb/c mice. Photochem. Photobiol. 73, 432-438.

11. Reeve, V. E., M. Bosnic, C. Boehm-Wilcox, N. Nishimura and R. D. Ley (1998) Ultraviolet A radiation $(320-400 \mathrm{~nm})$ protects hairless mice from immunosuppression induced by ultraviolet B radiation $(280-320 \mathrm{~nm})$ or cis-urocanic acid. Int. Arch. Allergy Immunol. $115,316-322$.

12. Tuchinda, C., H. W. Lim, F. M. Strickland, E. A. Guzma' n and H. K. Wong (2007) Comparison of broadband UVB, narrowband UVB, broadband UVA and UVA1 on activation of apoptotic pathways in human peripheral blood mononuclear cells. Photodermatol. Photoimmunol. Photomed. 23, 2-9.

13. Ibuki, Y., Y. Komaki, G. Yang and T. Toyooka (2021) Long-wavelength UVA enhances UVB-induced cell death in cultured keratinocytes: DSB formation and suppressed survival pathway. Photochem Photobiol Sci 20, 639-652.

14. Tonolli, P. N., O. Chiarelli-Neto, C. Santacruz-Perez, H. C. Junqueira, I. S. Watanabe, F. G. Ravagnani, W. K. Martins and M. S. Baptista (2017) Lipofuscin generated by UVA turns keratinocytes photosensitive to visible light. J. Invest. Dermatol. 137, 2447-2450.

15. Dreaden, T. M., J. Chen, S. Rexroth and B. A. Barry (2011) N-formylkynurenine as a marker of high light stress in photosynthesis. J. Biol. Chem. 286, 22632-22641.

16. Park, S. L., R. Justiniano, J. D. Williams, C. M. Cabello, S. Qiao and G. T. Wondrak (2015) The tryptophan-derived endogenous aryl hydrocarbon receptor ligand 6formylindolo[3,2-b]carbazole is a nanomolar UVA photosensitizer in epidermal keratinocytes. J. Invest. Dermatol. 135, 1649-1658.

17. Walrant, P. and R. Santus (1974) N-formyl-kynurenine, a tryptophan photooxidation product, as a photodynamic sensitizer. Photochem. Photobiol. 19, 411-417.

18. Douki, T. and E. Sage (2015) Dewar valence isomers, the third type of environmentally relevant DNA photoproducts induced by solar radiation. Photochem. Photobiol. Sci. 15, 24-30.

19. Taylor, J.-S. and M. P. Cohrs (1987) DNA, light, and Dewar pyrimidones: the structure and biological significance of TpT3. J. Am. Chem. Soc. 109, 2834-2835.

20. Escher, B. I., J. Hackermuller, T. Polte, S. Scholz, A. Aigner, R. Altenburger, A. Bohme, S. K. Bopp, W. Brack, W. Busch, M. Chadeau-Hyam, A. Covaci, A. Eisentrager, J. J. Galligan, N. Garcia-Reyero, T. Hartung, M. Hein, G. Herberth, A. Jahnke, J. Kleinjans, N. Kluver, M. Krauss, M. Lamoree, I. Lehmann, T. Luckenbach, G. W. Miller, A. Muller, D. H. Phillips, T. Reemtsma, U. Rolle-Kampczyk, G. Schuurmann, B. Schwikowski, Y. M. Tan, S. Trump, S. Walter-Rohde and J. F. Wambaugh (2017) From the exposome to mechanistic understanding of chemical-induced adverse effects. Environ. Int. 99, 97-106. 
21. Lasch, A., D. Lichtenstein, P. Marx-Stoelting, A. Braeuning and J. Alarcan (2020) Mixture effects of chemicals: The difficulty to choose appropriate mathematical models for appropriate conclusions. Environ. Pollut. 260, 113953.

22. Olmstead, A. W. and G. A. LeBlanc (2005) Toxicity assessment of environmentally relevant pollutant mixtures using a heuristic model. Integr. Environ. Assess. Manag. 1, 114-122.

23. Rider, C. V. and G. A. LeBlanc (2005) An integrated addition and interaction model for assessing toxicity of chemical mixtures. Toxicol Sci 87, 520-528.

24. Chou, T. C. and P. Talalay (1984) Quantitative-Analysis of Dose-Effect Relationships - the Combined Effects of Multiple-Drugs or Enzyme-Inhibitors. Adv. Enzyme Regul. 22, 27 55.

25. Chou, T. C. (2006) Theoretical basis, experimental design, and computerized simulation of synergism and antagonism in drug combination studies. Pharmacological Reviews 58, 621-681.

26. Chou, T. C. (2008) Preclinical versus clinical drug combination studies. Leuk. Lymphoma 49, 2059-2080.

27. Chou, T. C. (2011) The mass-action law based algorithm for cost-effective approach for cancer drug discovery and development. Am. J. Cancer Res. 1, 925-954.

28. Cadet, J. and T. Douki (2018) Formation of UV-induced DNA damage contributing to skin cancer development. Photochem. Photobiol. Sci. 17, 1816-1841.

29. Douki, T. (2006) Effect of denaturation on the photochemistry of pyrimidine bases in isolated DNA. J. Photochem. Photobiol. B: Biol. 82, 45-52.

30. Douki, T. (2016) Sunlight-induced DNA damage: molecular mechanisms and photoprotection strategies. In Skin Stress Response Pathways: Environmental Factors and Molecular Opportunities. (Edited by G. T. Wondrak), pp. 49-77. Springer Int. Publish.

31. Douki, T., L. Voituriez and J. Cadet (1991) Characterization of the (6-4) photoproduct of 2'-deoxycytidylyl-(3'-5')-thymidine and of its Dewar valence isomer. Photochem. Photobiol. 27, 293-297.

32. Taylor, J.-S., H.-L. Lu and J. J. Kotyk (1990) Quantitative conversion of the (6-4) photoproduct of TpdC to its Dewar valence isomer upon exposure to simulated sunlight. Photochem. Photobiol. 51, 161-167.

33. Lemaire, D. G. E. and B. P. Ruzsicska (1993) Quantum yields and secondary photoreactions of the photoproducts of dTpdT, dTpdC and dTpdU. Photochem. Photobiol. 57, 755769.

34. Douki, T. (2016) Relative contributions of UVB and UVA to the photoconversion of (6-4) photoproducts into their Dewar valence isomers. Photochem. Photobiol. 92, 587-594.

35. Douki, T. (2013) The variety of UV-induced pyrimidine dimeric photoproducts in DNA as shown by chromatographic quantification methods. Photochem. Photobiol. Sci. 12, 1286-1302.

36. Matallana-Surget, S., J. A. Meador, F. Joux and T. Douki (2008) Effect of the GC content of DNA on the distribution of UVB-induced bipyrimidine photoproducts. Photochem. Photobiol. Sci. 7, 794-801.

37. Douki, T., I. Berard, A. Wack and S. Andra (2014) Contribution of cytosine-containing cyclobutane dimers to DNA damage produced by photosensitized triplet-triplet energy transfer. Chem. Eur. J. 20, 5787-5794. 
38. Tuckerman, M. E. (2019) Chemical Kinetics - More complex reactions. In Physical chemistry for the biosciences. pp. 9.4.1-9.4.6. LibreTexts.

39. Courdavault, S., C. Baudouin, M. Charveron, A. Favier, J. Cadet and T. Douki (2004) Larger yield of cyclobutane dimers than 8 oxo-7,8-dihydroguanine in the DNA of UVAirradiated human skin cells. Mutat. Res. 556, 135-142.

40. Mouret, S., C. Baudouin, M. Charveron, A. Favier, J. Cadet and T. Douki (2006) Cyclobutane pyrimidine dimers are predominant DNA lesions in whole human skin exposed to UVA radiation. Proc. Natl. Acad. Sci. USA 103, 13765-13770.

41. Mouret, S., C. Philippe, J. Gracia-Chantegrel, A. Banyasz, S. Karpati, D. Markovitsi and T. Douki (2010) UVA-induced cyclobutane pyrimidine dimers in DNA: a direct photochemical mechanism? Org. Biomolec. Chem. 8, 1706-1711.

42. Johns, H. E., S. A. Rapaport and M. Delbück (1962) Photochemistry of thymine dimers. $J$. Mol. Biol. 4, 104-114.

43. Lemaire, D. G. E. and B. P. Ruzsicska (1993) Kinetic analysis of the deamination reactions of cyclobutane dimers of thymidylyl-3',5'-2'-deoxycytidine and 2'-deoxycytidine-3,5'thymidine. Biochemistry 32, 2525-2533.

44. Douki, T. and J. Cadet (2001) Individual determination of the yield of the main-UV induced dimeric pyrimidine photoproducts in DNA suggests a high mutagenicity of CC photolesions. Biochemistry 40, 2495-2501.

45. Clingen, P. H., C. F. Arlett, L. Roza, T. Mori, O. Nikaido and M. H. L. Green (1995) Induction of cyclobutane pyrimidine dimers, pyrimidine(6-4)pyrimidone photoproducts, and Dewar valence isomers by natural sunlight in normal human mononuclear cells. Cancer Res. 55, 2245-2248.

46. Qin, X. S., S. M. Zhang, M. Zarkovic, Y. Nakatsuru, S. Shimizu, Y. Yamazaki, H. Oda, O. Nikaido and T. Ishikawa (1996) Detection of ultraviolet photoproducts in mouse skin exposed to natural sunlight. Jap. J. Cancer Res. 87, 685-690.

47. Meador, J. A., A. J. Baldwin, J. D. Pakulski, W. H. Jeffrey, D. L. Mitchell and T. Douki (2014) The significance of the Dewar valence photoisomer as a UV radiation-induced DNA photoproduct in marine microbial communities. Environm. Microbiol. 16, 18081820.

48. Douki, T., T. Zalizniak and J. Cadet (1997) Far-UV-induced dimeric photoproducts in short oligonucleotides: Sequence effects. Photochem. Photobiol. 66, 171-179. 